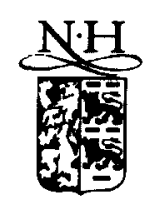

ELSEVIER
Physica D 102 (1997) 1-14

\title{
Bifocal homoclinic bifurcations
}

\author{
Carlo Laing *, Paul Glendinning ${ }^{1}$ \\ Department of Applied Mathematics and Theoretical Physics, University of Cambridge, Silver Street, Cambridge CB3 9EW, UK
}

Received 26 February 1996; revised 15 October 1996; accepted 20 October 1996

Communicated by J. Meiss

\begin{abstract}
Homoclinic orbits to bifocus-type stationary points have been studied theoretically by a number of authors, but up until now, only one analytic example has been found. In this paper we summarise and extend the known theory regarding bifocal homoclinic bifurcations and present numerical verification of some of the more interesting theoretical predictions that have been made.
\end{abstract}

Keywords: Homoclinic; Bifocal fixed point; Bifurcation; Codimension

\section{Introduction}

Homoclinic bifurcations have been studied by many authors during the past 30 years or so, e.g. [13,14,17], and it has become apparent that they can be thought of as "organising centres" for the dynamics of low-dimensional, deterministic dynamical systems. Much effort has gone into classifying the different sorts of bifurcation that can occur in terms of their genericity or typicality, and determining the sorts of behaviour that occur in systems that undergo these bifurcations.

Assuming that a vector field is generic, there are (up to time reversal) three possible types of homoclinic bifurcation involving hyperbolic stationary points: to a saddle, to a saddle-focus, and to a bifocus. The first two cases have been theoretically and numerically studied by a number of people and are well understood, whilst the last case has been studied theoretically but not numerically. This paper presents a numerical investigation of bifocal homoclinic bifurcations and verifies some of the theoretical predictions that have been made.

The rest of this paper is organised as follows: the remainder of this section introduces the idea of a homoclinic orbit and briefly summarises the known results for the saddle and saddle-focus case. Section 2 is a presentation of the known theoretical results for the bifocal case, with an emphasis on aspects that differ from the two cases mentioned above. Results are presented in the context of both codimension one and codimension two homoclinic

\footnotetext{
* Corresponding author. Tel.: +44 1223 337900; fax: +44 1223 337918; e-mail: c.r.laing@damtp.cam.ac.uk.

${ }^{1}$ Permanent address: School of Mathematical Sciences, Queen Mary and Westfield College, Mile End Road, London E1 4NS, UK.
} 
bifurcations. Section 3 is a short description of the technique used to construct a vector field that undergoes a bifocal homoclinic bifurcation. This has been presented elsewhere [11,12] and is thus brief. Section 4 presents numerical verification of some of the theoretically derived results presented in Section 2, while Section 5 is a summary that also gives some conjectures and ideas for further work.

A homoclinic orbit for an autonomous ordinary differential equation

$$
\dot{x}=f(x, \mu), \quad f: \mathbb{R}^{n} \times \mathbb{R} \mapsto \mathbb{R}^{n}
$$

is a non-trivial solution $x_{h}(t)$ of (1) that tends to a stationary point, $\bar{x}$, in both forwards and backwards time, i.e.

$$
\lim _{t \rightarrow \pm \infty} x_{h}(t)=\bar{x}, \quad x_{h}(0) \neq \bar{x}
$$

For typical vector fields the existence of a homoclinic orbit is non-generic and we expect there to be an isolated value of $\mu$, which from now on we assume to be 0 , at which the homoclinic orbit exists. We are normally interested in the possible dynamics of the vector field for small values of $|\mu|$.

Assuming that the homoclinic orbit does not pass too close to other stationary points in the flow, the local dynamics can be determined solely from the eigenvalues of the Jacobian of the vector field evaluated at the stationary point, $D f(\bar{x}, 0)$ (henceforth called $A$ ). Typically, trajectories which approach the stationary point as $t \rightarrow \infty$ do so tangential to the eigenspace spanned by the eigenvector(s) of $A$ corresponding to the eigenvalue(s) whose real part is negative and smallest in absolute value (which we refer to as the negative leading eigenvalue(s)), and those that approach the stationary point as $t \rightarrow-\infty$ do so tangential to the eigenspace spanned by the eigenvector(s) corresponding to the eigenvalue(s) whose real part is positive and smallest in numerical value (which we similarly call the positive leading eigenvalue(s)). Because of this behaviour in phase space, the values and signs of all non-leading eigenvalues are irrelevant to the dynamics for small $|\mu|$ in typical systems.

We assume that $A$ has no eigenvalues with zero real part, and that none of the real parts of the leading eigenvalues are repeated (apart from those of complex conjugate pairs, which necessarily are), both generic assumptions. Given this, there are (up to time reversal) three possible cases:

1. The leading eigenvalues of $A$ are real, viz. $\{\lambda, \nu\}$, where $\lambda<0<\nu$. This is known as the saddle case.

2. The leading eigenvalues are one real and one complex conjugate pair, viz. $\left\{\lambda \pm i \omega_{1}, v\right\}$, where $\lambda<0<v$ and $\omega_{1}>0$. This is known as a saddle-focus.

3. The leading eigenvalues are two complex conjugate pairs, viz. $\left\{\lambda \pm i \omega_{1}, v \pm i \omega_{2}\right\}$, where $\lambda<0<v$ and $\omega_{1}, \omega_{2}>0$. This is known as a bifocus.

Note that the dimension of phase space (the $n$ in Eq. (1)) must be greater than 1 for case 1 to occur, greater than 2 for case 2, and greater than 3 for case 3 .

For case 1 (the saddle), under the generic assumption that $\lambda+\nu \neq 0$, a periodic orbit is either created or destroyed as $\mu$ passes through 0 . As $\mu$ tends to zero from the appropriate side, the periodic orbit tends to the homoclinic orbit and the period of the orbit tends to infinity.

For case 2 (the saddle-focus) the dynamics for $|\mu|$ small depends on whether the ratio $|\lambda / \nu|$ is greater than or less than 1 . If this ratio (henceforth known as $\delta$ ) is greater than 1, the dynamics is essentially the same as case 1: a periodic orbit is created or destroyed as $\mu$ passes through 0 and the period of the orbit tends to infinity as $\mu$ tends to 0 .

If, however, $\delta<1$, the differential equation has chaotic solutions for arbitrarily small values of $|\mu|$, and there are an infinite number of saddle-node bifurcations of periodic orbits and period-doubling bifurcations in any $\mu$-interval containing the origin. There are also an infinite number of $\mu$-values accumulating on 0 from one side at which there are "double-pulse" homoclinic orbits that make two large excursions away from the stationary point before 

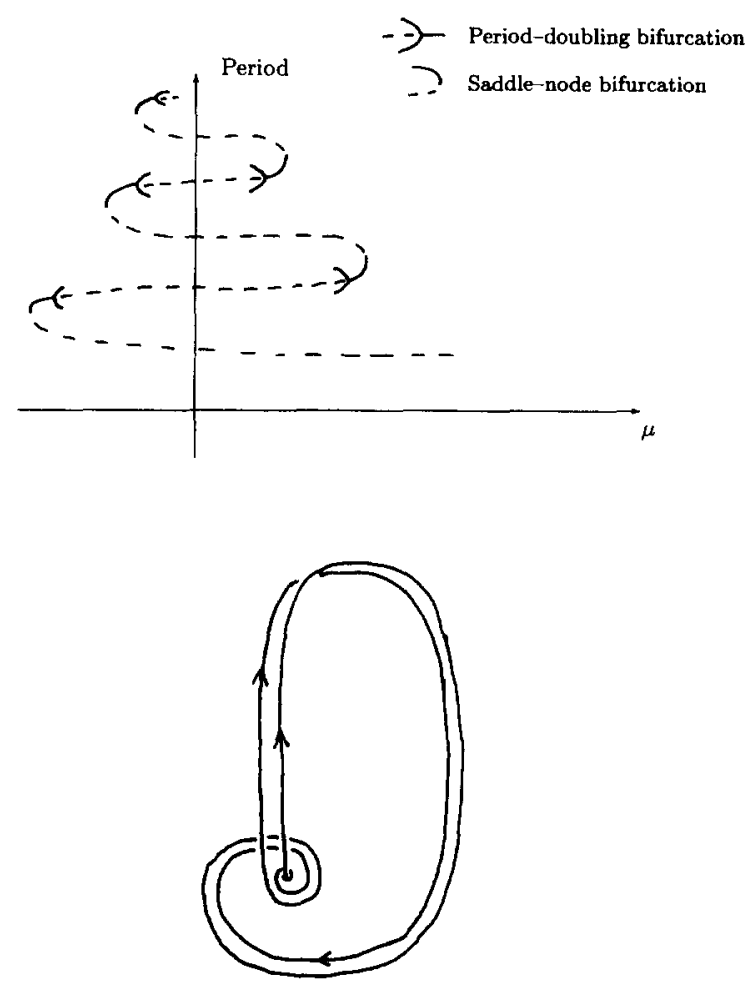

Fig. 1. Top: a schematic sketch of period versus $\mu$ for the saddle-focus case showing some of the infinite number of saddle-node and period-doubling bifurcations that accumulate on $\mu=0$. Bottom: A schematic sketch of a "double-pulse" homoclinic orbit to a saddle-focus.

approaching it. These last two concepts are illustrated schematically in Fig. 1. (Note the stabilty of the periodic orbit in the top sketch - there are period-doubling bifurcations on every second "wiggle".)

It can be shown that the asymptotic (as period $\rightarrow \infty$ ) difference in period between successive crossings of the $\mu=0$ line in Fig. 1 (top) is $\pi / \omega_{1}$, while the ratio of successive values of $\mu$ at which saddle-node bifurcations of periodic orbits occur is $-\exp \left(-\pi \nu / \omega_{1}\right)$, where the initial minus sign is due to the oscillation of the curve about $\mu=0$.

These results have been derived by a number of authors; see for example $[9,13,15]$.

\section{Theory of bifocal homoclinic bifurcations}

The existence of countably many periodic orbits when $\mu=0, n=4$ and there is a homoclinic orbit to a bifocus was proved by Shil'nikov [14] in 1967, and this result was extended by him in 1970 to show that when $\mu=0$ there is an uncountable number of aperiodic orbits in either the saddle-focus or bifocus case [15]. Bifurcations that occur when a homoclinic orbit to a bifocus is perturbed were analysed in a theoretical sense in [8]. Two papers that investigate double-pulse bifocal homoclinic orbits are Refs. $[1,10]$, while Refs. $[11,12]$ show numerical evidence for the existence of bifocal homoclinic orbits in specific examples. Section 2.1 is a summary of codimension one phenomena derived in $[1,8,10]$, and is not intended as a presentation of new results, while Section 2.2, regarding codimension two behaviour, does contain some new results. 


\subsection{Codimension one phenomena}

As mentioned above, it was shown by Shil'nikov [15] that if $\mu=0$, there is chaotic behaviour in an arbitrarily small neighbourhood in phase space of a bifocal homoclinic orbit. Fowler and Sparrow [8] showed that a plot of period versus $\mu$ has the same shape as that shown at the top of Fig. 1 (though the position of period-doubling bifurcations and the stability of the orbits could not be determined) and also derived some scaling results for the shape of the curve. As in the saddle-focus case, we will define a parameter $\delta$ as $\delta \equiv|\lambda / \nu|$, where $\lambda$ and $\nu$ are as in case 3 of Section 1. The asymptotic difference in period between successive crossings of the $\mu=0$ line depends on whether $\delta$ is greater or less than 1. If $\delta>1$, the spacing is $\pi / \omega_{2}$, whereas if $\delta<1$, it is $\pi / \omega_{1}$. The asymptotic ratio of successive values of $\mu$ at which there are saddle-node bifurcations of periodic orbits also depends on the magnitude of $\delta$. If $\delta>1$, the ratio is $-\exp \left(-\nu \pi / \omega_{2}\right)$, whereas if $\delta<1$, it is $-\exp \left(\lambda \pi / \omega_{1}\right)$.

This change in scaling reflects the symmetry between the $\delta<1$ and $\delta>1$ cases, which can be transformed from one to the other by time reversal.

It was shown in $[1,10]$ that double-pulse homoclinic orbits can exist near a bifocal stationary point, but in contrast with the saddle-focus case (assuming a certain non-resonance condition on $\omega_{1}$ and $\omega_{2}$ ), the values of $\mu$ at which they occur accumulate on $\mu=0$ from both sides, with the exact form of the accumulation depending on the ratio $\omega_{1} / \omega_{2}$. If the resonance condition $\omega_{1} / \omega_{2}=2 n$ for some $n \in \mathbb{Z}^{+}$holds, the values of $\mu$ at which there are double-pulse homoclinic orbits accumulate on 0 from one side only.

Recall that a periodic orbit in $\mathbb{R}^{n}$ has associated with it $n-1$ non-trivial Floquet multipliers, and that the product of these must be positive. One consequence of this is that if a periodic orbit in $\mathbb{R}^{3}$ undergoes a saddle-node bifurcation (multiplier passing through +1 ) and then a period-doubling bifurcation (multiplier passing through -1 ), it must undergo reverse period-doubling before undergoing another saddle-node bifurcation. This is precisely what happens on every second branch in Fig. 1 (top). However, this no longer holds in $\mathbb{R}^{4}$ and it is quite plausible that a periodic orbit in $\mathbb{R}^{4}$ involved in a homoclinic bifurcation to a bifocus undergoes a period-doubling bifurcation and then a number of intervening saddle-node bifurcations before reverse period-doubling, as conjectured in [8] and illustrated in Fig. 22 of that paper.

\subsection{Codimension two phenomena}

In a generic dissipative system, a homoclinic bifurcation is a codimension one phenomenon; if some other non-generic condition also holds, the bifurcation is of codimension two. A number of bifurcations of this type are catalogued in [6]. We will introduce three more involving bifocal homoclinic bifurcations and mention two from [6].

Apart from the bifurcation parameter $\mu$, the behaviour near a bifurcation to a bifocus depends largely on two other parameters, viz. the ratios $\delta$ and $\omega_{1} / \omega_{2}$. The three possible codimension two bifurcations occur when, as well as having a homoclinic bifurcation,

1. $\delta=1$ while $\omega_{1} / \omega_{2}$ is bounded away from both 0 and $\infty$, or

2. $\omega_{1} / \omega_{2}=0$ while $\delta<1$, or

3. $\omega_{1} / \omega_{2}=0$ while $\delta>1$.

In the last two cases, the Jacobian at the stationary point has a repeated real eigenvalue of $\lambda$. Unfoldings of this will produce either a complex conjugate pair, so that the stationary point is a bifocus, or a pair of unequal real eigenvalues, so that it is a saddle-focus.

It may be helpful to look forward to Fig. 2. In this, $\epsilon$ controls the unfolding of the repeated eigenvalue mentioned above. When $\epsilon>0$, the stationary point is a bifocus, while for $\epsilon<0$, it is a saddle-focus. Thus, the three cases above correspond to moving along paths 1,2 and 3, respectively, in this figure. The transition that occurs along path 4 is mentioned in [6], and has been studied by Belyakov [4] and Bernoff [5]. Since the stationary point is not 


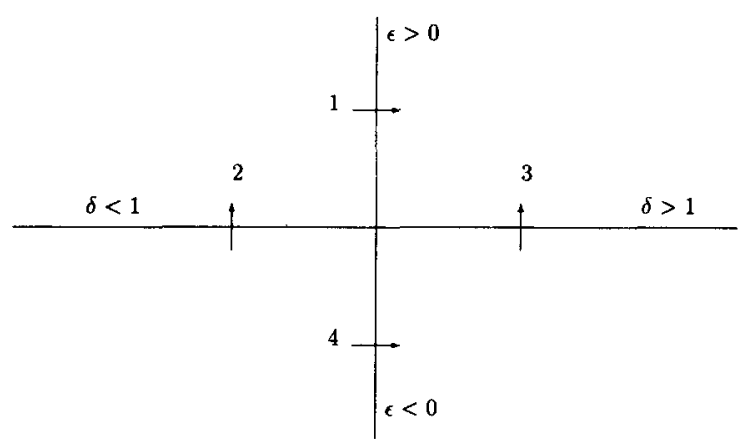

Fig. 2. Four possible paths in $(\epsilon, \delta)$ space along which codimension two bifurcations occur.

a bifocus at any point along this path, we will not mention it further. Paths 2 and 3 are discussed below, while path 1 is mentioned in the conclusion.

\subsubsection{Path 2}

Along path 2, we expect there to be an infinite number of saddle-node and period-doubling bifurcations accumulating on $\mu=0$ for $\epsilon$ both positive and negative, i.e. there to be no qualitative change in this aspect as we pass through the codimension two point in $(\mu, \epsilon)$ space.

The only significant change is due to the behaviour of the double-pulse homoclinic orbits. If $\epsilon<0$, parameter values at which these occur accumulate on 0 from one side only, whereas if $\epsilon>0$, they can accumulate from both sides. Given this, we might expect to see curves in $(\mu, \epsilon)$ space on which there are bifocal homoclinic orbits move from one side of $\mu=0$ to the other as $\epsilon$ is increased through zero.

\subsubsection{Path 3}

Along path 3 we expect a significant change as we move through the codimension two point, since if $\epsilon<0$ there are no saddle-node bifurcations of periodic orbits, whereas if $\epsilon>0$ there is an infinite number accumulating on $\mu=0$. Belyakov [3] studied a similar problem (the transition from a saddle to a saddle-focus) and derived an equation for the asymptotic shape of the curves of saddle-node bifurcations of periodic orbits that emanate from $(\mu, \epsilon)=(0,0)$ for $\epsilon>0$. An essentially equivalent result can be derived for the saddle-focus to bifocus transition. Below, we outline both Belyakov's result and the result for the saddle-focus to bifocus transition.

\subsection{Belyakov's result}

Assume that the linearisation of the flow about the stationary point is

$$
\dot{r}=\lambda r, \quad \dot{\theta}=\omega_{1}, \quad \dot{z}=\nu z,
$$

where $\lambda<0<v$ and $\omega_{1}>0$, and that there is a homoclinic connection to the stationary point at $\mu=0$. By considering the flow near the stationary point and using the global reinjection, we obtain an approximate map from a return plane to itself. This map is two-dimensional, but by assuming that we are close to the homoclinic orbit 
in both phase and parameter space, we can approximate the problem of finding periodic orbits in the flow by the problem of finding solutions of the equation

$$
z-\mu \approx \beta z^{\delta} \cos \left[\frac{\omega_{1}}{v}(\log h-\log z)+\phi_{2}\right],
$$

where $\beta(>0), h$ and $\phi_{2}$ are constants relating to the exact geometry of the flow and $\delta=|\lambda / \nu|$ (see for example [13] for a derivation of this result).

Each root of this equation approximately corresponds to a periodic orbit in the flow, the approximation getting better as the homoclinic orbit is approached.

In the following, we assume that $\delta$ and $v$ are fixed, impose the condition that there is a saddle-node bifurcation of periodic orbits in the flow, and then find the shape of the curve in $\left(\mu, \omega_{1}\right)$ space on which this occurs. There are an infinite number of these curves, which we label with $n$ in the obvious way ( $n \in \mathbb{Z}$ ); curves corresponding to successive values of $n$ lie on opposite sides of $\mu=0$.

For $|\mu|$ small enough, the condition for a saddle-node bifurcation to occur is approximately

$$
\frac{\mathrm{d}}{\mathrm{d} z}\left\{\beta z^{\delta} \cos \left[\frac{\omega_{1}}{v}(\log h-\log z)+\phi_{2}\right]\right\}=1,
$$

where this derivative is evaluated at a solution of (2). Eq. (3) is equivalent to

$$
-\lambda \cos \left[\frac{\omega_{1}}{\nu}(\log h-\log z)+\phi_{2}\right]+\omega_{1} \sin \left[\frac{\omega_{1}}{v}(\log h-\log z)+\phi_{2}\right]=\frac{v}{\beta z^{\delta-1}} .
$$

Since $\delta<1$ and we are interested in the limit $z \rightarrow 0$, we replace the right-hand side of (4) with zero, resulting in the equation

$$
\tan \left[\frac{\omega_{1}}{\nu}(\log h-\log z)+\phi_{2}\right] \approx \frac{\lambda}{\omega_{1}} .
$$

We also know that solutions of (2) are created and destroyed near the extrema of the right-hand side of (2), and we approximate this by saying that appropriate values of $z$ at which to evaluate (3) are roots of the equation

$$
z-\mu \approx \pm \beta z^{\delta}
$$

Again, since $\delta<1$ and we are interested in the limit $z \rightarrow 0$, roots of this are approximately

$$
z_{ \pm} \approx\left(\frac{\mp \mu}{\beta}\right)^{1 / \delta}
$$

Substituting these values of $z$ into (5) gives

$$
\tan \left[\frac{\omega_{1}}{v} \log h+\frac{\omega_{1}}{\lambda} \log \left(\frac{\mp \mu}{\beta}\right)+\phi_{2}\right] \approx \frac{\lambda}{\omega_{1}}
$$

and for this to hold as $\omega_{1} \rightarrow 0$, we need $\left(\omega_{1} / \lambda\right) \log (\mp \mu / \beta)+\phi_{2}$ to tend to $\left(n+\frac{1}{2}\right) \pi$ for some $n \in \mathbb{Z}$ in this limit. This rearranges to

$$
\mu_{n} \sim(-1)^{n} B \exp \left(-k_{n} / \omega_{1}\right),
$$

where $k_{n}=-\lambda\left[\left(n+\frac{1}{2}\right) \pi-\phi_{2}\right]$ and $B$ is a constant. (Note that for $(9)$ to make sense, $n$ must be greater than some positive $n_{0}$.) This is the appropriate curve of saddle-node bifurcations of periodic orbits in $\left(\mu, \omega_{1}\right)$ space, and holds in the limit $\left(\mu, \omega_{1}\right) \rightarrow(0,0)$. Note also that although the values of $k_{n}$ depend on the problem-specific quantity $\phi_{2}$, $\left|k_{n}-k_{n+1}\right|=\lambda \pi$, a quantity we assumed to be constant in our derivation. 


\subsection{The saddle-focus to bifocus transition}

In a similar way to the above, assume that the linearisation of the flow about the stationary point is

$$
\dot{r}_{1}=\lambda r_{1}, \quad \dot{\theta}_{1}=\omega_{1}, \quad \dot{r_{2}}=v r_{2}, \quad \dot{\theta}_{2}=\omega_{2},
$$

where $\lambda<0<v$ and $\omega_{1}, \omega_{2}>0$, and that there is a homoclinic connection when $\mu=0$. Then the following map for successive values of $r_{2}$, derived as Eq. (4.2) in [8], gives approximate behaviour of orbits in the flow:

$$
r_{2}^{i+1} \cos \left(-\frac{\omega_{2}}{v} \log \left(k / r_{2}^{i+1}\right)+\phi_{2}\right)-\beta\left(r_{2}^{i}\right)^{\delta} \cos \left(-\frac{\omega_{1}}{\lambda} \log \left(k / r_{2}^{i}\right)+\phi_{1}\right)=\mu
$$

The quantities $\beta, k, \phi_{1}$ and $\phi_{2}$ are constants which depend on the exact geometry of the flow. Recall that $\delta=|\lambda / \nu|>$ 1. Fixed points of (10) approximately correspond to periodic orbits in the flow. Since $\delta>1$ and $\beta$ is of order 1 , and we are interested in the $r_{2} \rightarrow 0$ limit, the second term on the left-hand side of (10) can be neglected relative to the first one, i.e. to lowest order, the equation we are interested in is

$$
r \cos \left(-\frac{\omega_{2}}{v} \log (k / r)+\phi_{2}\right) \approx \mu
$$

where we have dropped the sub- and superscripts on $r$. The assumption that there is a saddle-node bifurcation in the flow is approximately equivalent to either

$$
\left[\frac{-\omega_{2}}{\nu} \log (k / r)+\phi_{2}\right]_{r=\mu}=2 n \pi \quad \text { if } \mu>0
$$

or

$$
\left[\frac{-\omega_{2}}{v} \log (k / r)+\phi_{2}\right]_{r=-\mu}=(2 n+1) \pi \quad \text { if } \mu<0 .
$$

Rearranging these leads to

$$
\mu_{n} \sim(-1)^{n} k \exp \left(-b_{n} / \omega_{2}\right),
$$

where $b_{n}=v\left(\phi_{2}-n \pi\right)$ and $k$ is a constant. (In a similar way to above, $n$ must be less than some negative $n_{0}$.) Compare this with Eq. (9). Although (9) and (12) are of the same form, there is an important difference - Eq. (9) was derived under the assumption that $\omega_{1} \rightarrow 0$, and is only valid in this limit, while the derivation of (12) did not assume the limit $\omega_{2} \rightarrow 0$. The fact that Eqs. (9) and (12) are so similar is perhaps not surprising, because (as mentioned in [8]) Eq. (10) is close to Eq. (2) when $\delta$ is far from one. In a similar way to the saddle to saddle-focus transition, $\left|b_{n}-b_{n+1}\right|=\pi v$ in Eq. (12).

\section{Construction of examples}

This section is a short presentation of the technique used to construct a vector field that has a bifocal homoclinic orbit. It has been described elsewhere in more detail $[11,12]$. The basic idea is to take a three-dimensional vector field which has a homoclinic orbit to a saddle-focus and augment the phase space by one dimension in such a way that the homoclinic connection is preserved and that the stationary point changes from a saddle-focus to a bifocus.

Assume we have a system

$$
\dot{X}=\left(\begin{array}{c}
\dot{x} \\
\dot{y} \\
\dot{z}
\end{array}\right)=A X+f(X, \mu)
$$


(where $\boldsymbol{X} \in \mathbb{R}^{3}, f: \mathbb{R}^{3} \times \mathbb{R} \mapsto \mathbb{R}^{3}$ contains only nonlinear terms, $\mu$ is a scalar bifurcation parameter and $\boldsymbol{A}$ is a $3 \times 3$ matrix with eigenvalues $\lambda \pm i \omega_{1}, v$, where $\lambda<0<v$ ) which has a homoclinic orbit to the origin at $\mu=0$. Then the augmented system

$$
\dot{\boldsymbol{X}}=\boldsymbol{A X}+\boldsymbol{f}(\boldsymbol{X}, \mu)-w e_{3}, \quad \dot{w}=\epsilon\left(\boldsymbol{e}_{3}^{\dagger} \cdot \boldsymbol{X}\right) / D_{3}+\nu w
$$

(where $\boldsymbol{e}_{3}, \boldsymbol{e}_{3}^{\dagger}$ are, respectively, the eigenvector and adjoint eigenvector of $A$ corresponding to $v, D_{3}=\boldsymbol{e}_{3}^{\dagger} \cdot \boldsymbol{e}_{3}$ and $w \in \mathbb{R}$ ) has eigenvalues at the origin of $\lambda \pm \mathrm{i} \omega_{1}$ and $v \pm \sqrt{-\epsilon}$. Since homoclinic orbits are a codimension one phenomenon, we expect there to be a curve with $\epsilon$ small and positive and $|\mu|$ small along which (14) has a homoclinic orbit to the origin, which is necessarily a bifocus. It may be useful to refer again to Fig. 2 , where the connection between $\epsilon$ and the $\omega_{2}$ of Section 2.2 is now more clear, i.e. $\omega_{2}=\sqrt{\epsilon}$ when $\epsilon>0$.

This technique was implemented in $[11,12]$ and numerical evidence for the existence of bifocal homoclinic orbits was shown there.

\section{Numerical verification of theory}

The numerical results in the remainder of this paper were obtained from a system constructed as in Section 3 . The system used as (13) is one studied by Arnéodo et al. [2]:

$$
\dot{x}=y, \quad \dot{y}=z, \quad \dot{z}=-x^{2}+\alpha x-\gamma y-z,
$$

where $\alpha$ and $\gamma$ are scalar parameters. Arnéodo et al. set $\gamma$ to various values in order to fix the value of $\delta$ at homoclinicity, and used $\alpha$ as the bifurcation parameter. For $0.4<\gamma<3$, there is a unique positive value of $\alpha$ at which there is a homoclinic connection to the origin, which is a saddle-focus. The value of $\delta$ at the origin decreases through 1 as $\gamma$ is increased through $\gamma_{1} \approx 1.3$.

There are two slightly different ways to augment this system, depending on exactly which features one is interested in, and these are outlined below.

\subsection{Path 2}

In this section, we simply calculate the eigenvalues and eigenvectors of the Jacobian of (15) at the origin and write down the equivalent of (14):

$$
\begin{aligned}
& \dot{x}=y-w \\
& \dot{y}=z-v w \\
& \dot{z}=-x^{2}+\alpha x-\gamma y-z-v^{2} w \\
& \dot{w}=\epsilon\left[\frac{\alpha v x+(\alpha-\gamma v) y+v^{2} z}{v^{4}+\gamma v^{2}+2 \alpha \nu}\right]+v w
\end{aligned}
$$

where $\nu$ is the positive real root of $s^{3}+s^{2}+\gamma s-\alpha=0$. If we set $\gamma$ to 2 , the value of $\delta$ at homoclinicity is greater than 1 for $|\epsilon|$ small, and we can verify the expected behaviour along path 2 . As mentioned in Section 2.2.1, we might expect to see the curves on which there are bifocal homoclinic orbits change from one side of $\alpha_{h}$ to the other as $\epsilon$ is increased through zero ( $\alpha_{h}$ being the value of $\alpha$ in Eq. (16) at which there is a homoclinic bifurcation). This has indeed been observed, and some results are shown in Figs. 3 and 4.

(All numerical results in this paper were obtained using the software package AUTO [7]. The curves of "homoclinic bifurcations" shown in the figures were obtained by computing the locus of periodic orbits with very high period. 


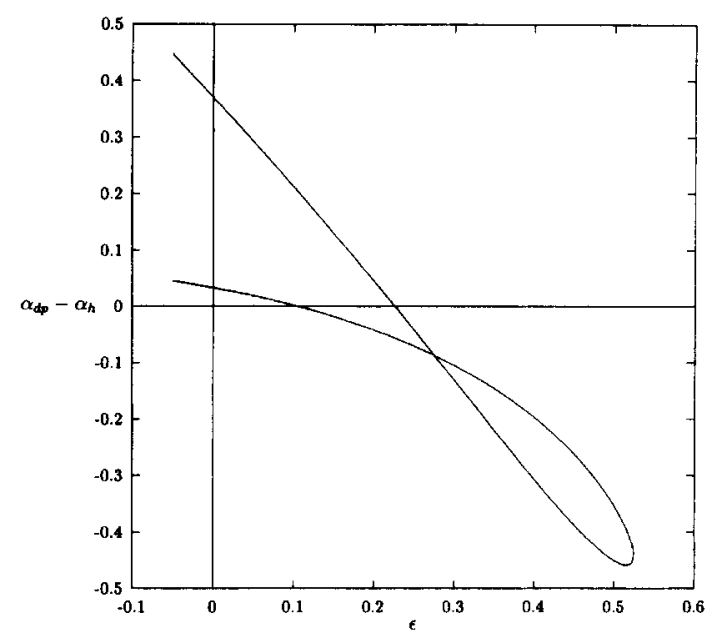

Fig. 3. The relative positions of two curves of double-pulse homoclinic bifurcations and the curve of primary homoclinic bifurcations for Eq. (16). The horizontal axis is $\epsilon$ and the vertical one is the difference between the $\alpha$-values for the two curves $\left(\alpha_{\mathrm{dp}}\right)$ and $\alpha_{\mathrm{h}}$. Two crossings are clearly seen as $\epsilon$ increases. Note that in this case the two curves of double pulse homoclinics join together as $\epsilon$ increases. (The curves of "homoclinic bifurcations" are actually the locus of orbits with very high period, as explained in the text.)

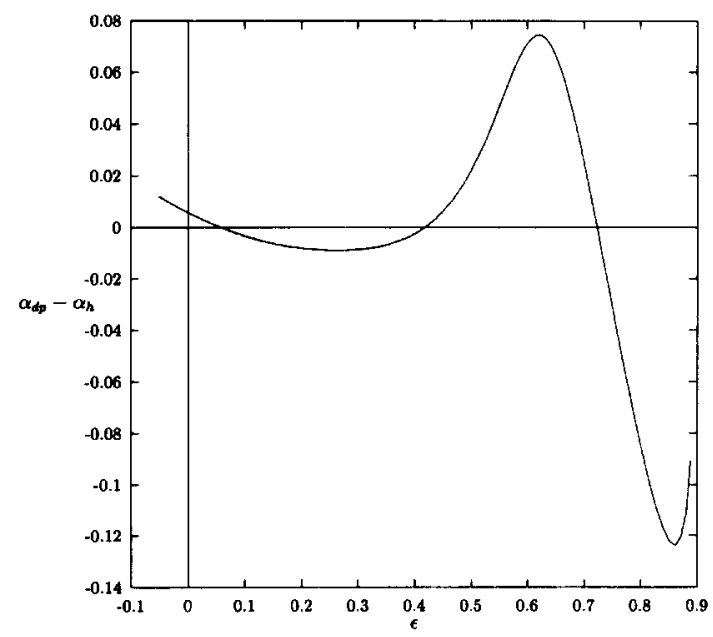

Fig. 4. The relative position of one curve of double-pulse homoclinic bifurcations and the curve of primary homoclinic bifurcations for Eq. (16). The horizontal axis is $\epsilon$ and the vertical is the difference between the $\alpha$-value for the curve and $\alpha_{h}$.

We could have performed numerical experiments as in [12] to provide more convincing proof of the existence of homoclinic orbits, but have taken the results from AUTO as sufficient.)

\subsection{Path 3}

In this section we wish to verify the theoretical results from Section 2.2.2. To do this we require all the parameters of the unaugmented system to be constant, so that we can vary $\epsilon$ and $\mu$ and verify Eq. (12). 


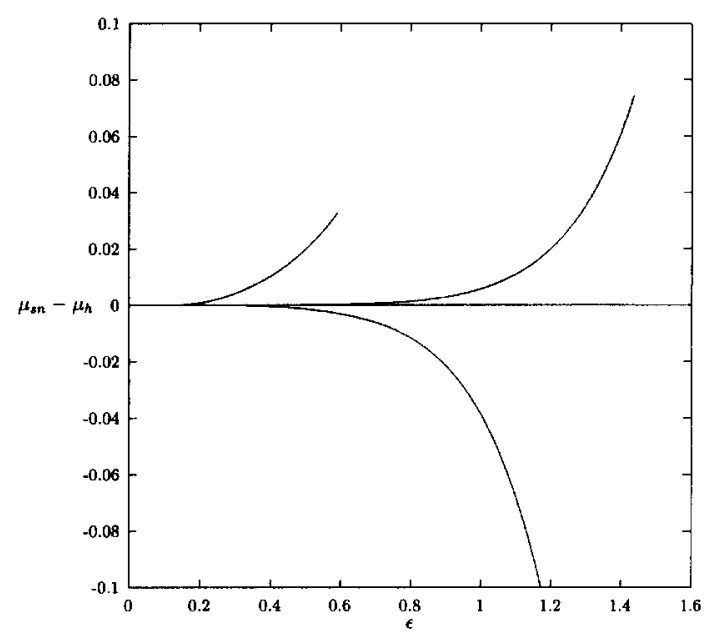

Fig. 5. The positions of three successive curves of saddle-node bifurcations of periodic orbits relative to the curve of primary homoclinic bifurcations for Eq. (17). The horizontal axis is $\epsilon$ and the vertical is the difference in $\mu$-values between the saddle-node curves and the homoclinic curve.

If $\gamma=0.5$ in (15), there is a principle homoclinic bifurcation to the origin when $\alpha \approx 0.9641494$, and in this case, the eigenvalues of the Jacobian evaluated at the origin are approximately $-0.815373 \pm 0.929383 \mathrm{i}$ and 0.630745854 , and the eigenvector and adjoint eigenvector corresponding to the real eigenvalue are approximately

$$
\left(\begin{array}{c}
0.801639004 \\
0.505630478 \\
0.318924328
\end{array}\right) \text { and }\left(\begin{array}{c}
0.624255617 \\
0.6659763565 \\
0.40838758217
\end{array}\right) \text {, }
$$

respectively. Using these vectors to augment $(15)$ when $(\alpha, \gamma)=(0.9641494,0.5)$ and introducing a bifurcation parameter $\mu$ results in the system:

$$
\begin{aligned}
\dot{x} & =y-0.801639004 w \\
\dot{y} & =z-\mu x^{2}-0.505630478 w \\
\dot{z} & =0.9641494 x-x^{2}-0.5 y-z-0.318924328 w \\
\dot{w} & =\epsilon\left[\frac{0.624255617 x+0.6659763565 y+0.40838758217 z}{0.9674103297}\right]+0.630745854 w .
\end{aligned}
$$

Note that while these coefficients have been calculated only approximately, it was exactly Eq. (17) that was numerically studied.

As explained in Section 2.2.2, we expect to see curves of saddle-node bifurcations of periodic orbits emanating from $(\mu, \epsilon)=(0,0)$ along path 3 and for them to have a shape given by (12). Three such (successive) curves for (17) are shown in Fig. 5, and a plot of $\log \left|\mu_{\mathrm{sn}}-\mu_{\mathrm{h}}\right|$ versus $1 / \sqrt{\epsilon}$ for these data is shown in Fig. 6. Although these curves are among the first few, and hence not expected to agree particularly well with asymptotic results, the average difference in slopes of successive curves is close to the expected value of $0.630745853 \pi \approx 2$. 


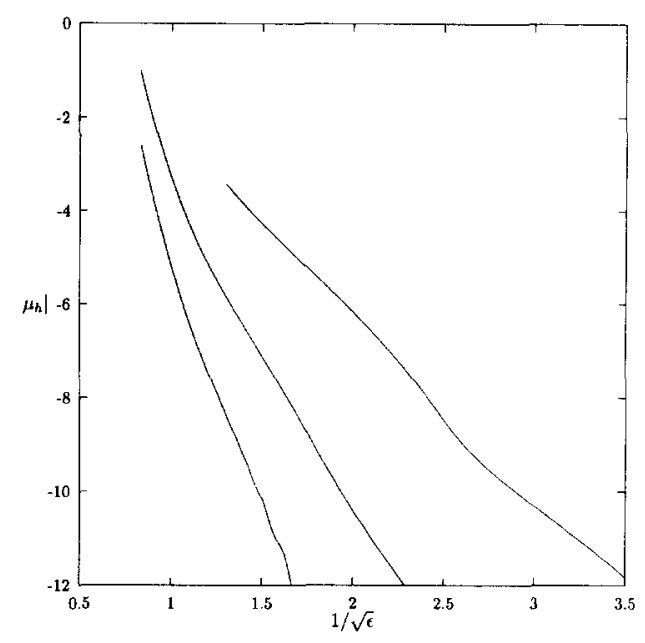

Fig. 6. The data points shows $\log \left|\mu_{\mathrm{sn}}-\mu_{\mathrm{h}}\right|$ versus $\epsilon^{-1 / 2}$ for the data in Fig. 5 .

\subsection{Belyakov's scaling}

This section is a numerical verification of the result derived by Belyakov [3] and described in Section 2.2 for the shape of the curves of saddle-node bifurcations of periodic orbits in the transition from a homoclinic orbit to a saddle-focus to a homoclinic orbit to a saddle. The system we study is the following

$$
\begin{aligned}
& \dot{x}=y-z, \\
& \dot{y}=2.657466 x+2.328733 y+x^{2}+x y+\mu x^{2}+0.83893461 z, \\
& \dot{z}=\epsilon\left[\frac{2.657466 x-0.83893461 y}{3.361277}\right]-0.83893461 z .
\end{aligned}
$$

This system was derived by augmenting a truncated unfolding of the normal form of the Takens-Bogdanov bifurcation

$$
\dot{x}=y, \quad \dot{y}=2 c x+(1+c) y+x^{2}+x y,
$$

which is numerically observed to have a homoclinic connection to the origin at $c \approx 1.328733$, in a way similar to that explained in Section 3. When $c=1.328733$, the Jacobian of (19) has eigenvalues of approximately 3.1676676 and -0.83893461 , and the eigenvector and adjoint eigenvector associated with the negative eigenvalue are approximately

$$
\left(\begin{array}{c}
1 \\
-0.83893461
\end{array}\right) \text { and }\left(\begin{array}{c}
2.657466 \\
-0.83893461
\end{array}\right)
$$

respectively. Hence the augmented system (18). When $\epsilon=z=0$, the system (18) restricted to the $(x, y)$-plane has a homoclinic connection to the origin at $\mu=0$. The eigenvalues of the Jacobian of the full three-dimensional system evaluated at the origin are $3.1676676,-0.83893461 \pm i \sqrt{\epsilon}$, when $\epsilon>0$; i.e. the $\omega_{1}$ of Eq. (9) is $\sqrt{\epsilon}$.

Four curves of saddle-node bifurcations of periodic orbits relative to the curve of homoclinic bifurcations for this augmented system are shown in Fig. 7. A plot of $\log \left|\mu_{\mathrm{sn}}-\mu_{\mathrm{h}}\right|$ as a function of $1 / \sqrt{\epsilon}$ for these curves is shown in Fig. 8. The average of the difference in slopes of successive curves is 2.67 , in good agreement with the expected value (from Section 2 ) of $|-0.83893461 \pi| \approx 2.64$. 


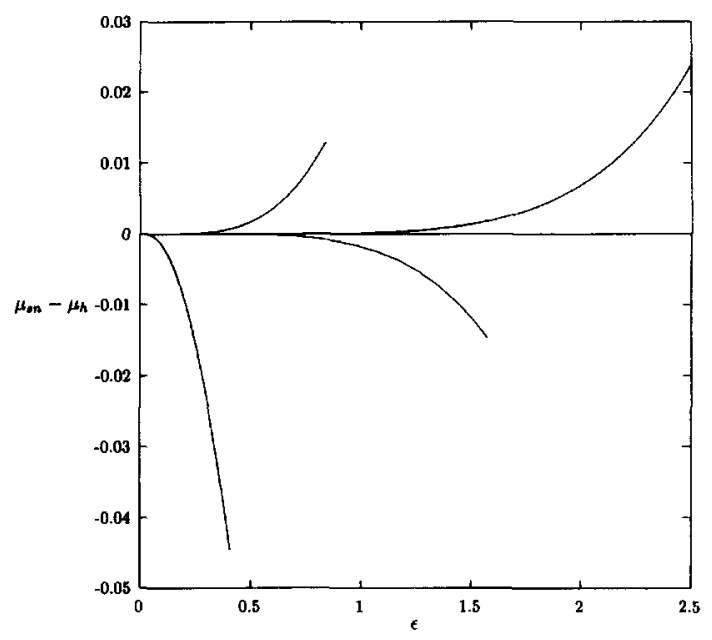

Fig. 7. A plot of the difference in $\mu$-values for four curves of saddle-node bifurcations of periodic orbits and the curve of homoclinic bifurcation as a function of $\epsilon$ for system (18).

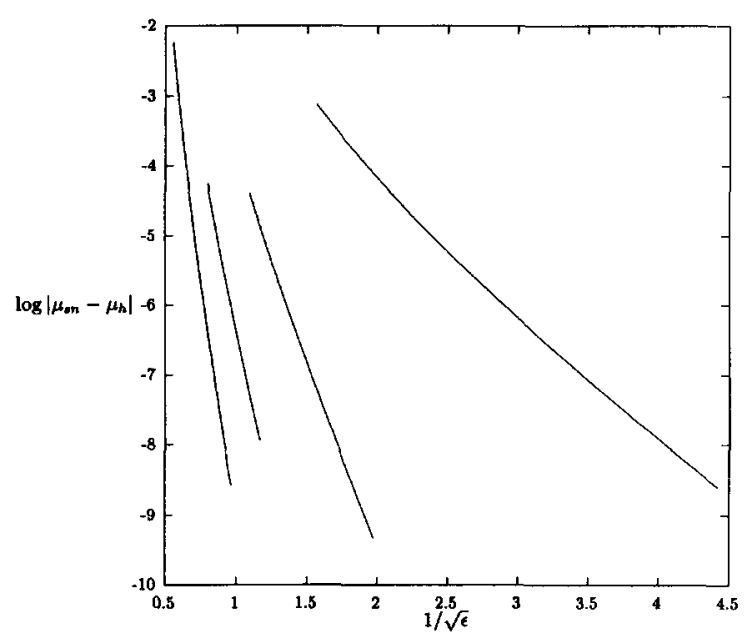

Fig. 8. A plot of $\log \left|\mu_{\mathrm{sn}}-\mu_{\mathrm{h}}\right|$ versus $\epsilon^{-1 / 2}$ for the data shown in Fig. 7.

\subsection{Codimension one phenonema}

The possibility of a periodic orbit involved in a bifocal homoclinic orbit undergoing period-doubling and then a number of saddle-node bifurcations before reverse period-doubling was conjectured in [8] and mentioned in Section 2.1. This behaviour has been observed in (16) for $\gamma=0.5$ and is illustrated in Fig. 9 - a plot of period and half-period (for the doubled orbit) versus $\alpha$ when $\epsilon=0.22346$. 


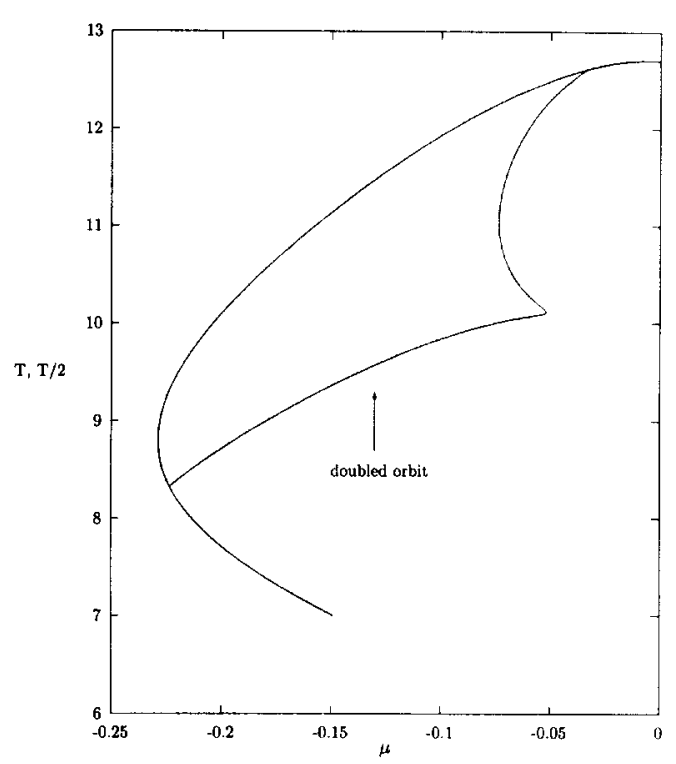

Fig. 9. A plot of period $T$ (of the basic orbit) and half-period $\frac{1}{2} T$ (of the period-doubled orbit) versus $\alpha$ for Eq. (16), showing the bifurcation sequence "period-doubling, saddle-node, reverse period-doubling". The value of $\epsilon$ is 0.22346 , and $\gamma$ is 0.5 .

\section{Conclusion and comments}

We did not dicuss path 1 of Fig. 2 in this paper. The bifurcations that occur along here are expected to be of interest because when $\delta=1$, the sum of the eigenvalues of the Jacobian is zero and the flow is then locally "conservative" in some sense. There are expected to be some similarities with the $|\lambda / \nu|=0.5$ case for the saddle-focus, as the flow in this case is also "divergence-free" at the stationary point. This last case is mentioned in [6], but does not seem to have been studied yet.

One other avenue open for investigation is the application of the technique discussed in Section 3 to systems which possess some symmetries. One example would be the Lorenz system, studied in [16], about which much is already known. It would then be possible to investigate (for example) heteroclinic orbits between two symmetric bifocal stationary points or symmetric pairs of orbits homoclinic to bifocal stationary points.

In this paper, we presented the known theoretical results regarding bifocal homoclinic bifurcations in the context of both codimension one and codimension two bifurcations, compared them with results for the saddle and saddlefocus case, and presented a new result regarding the transition from a saddle-focus homoclinic orbit to a bifocus homoclinic orbit. We then showed results from a numerical investigation of some of the more interesting aspects of the theory. There was good agreement between theory and numerical results.

\section{Acknowledgements}

CL is supported by the Cambridge Commonwealth Trust. 


\section{References}

[1] J.C. Alexander and C.K.R.T. Jones, Existence and stability of asymptotically oscillatory double pulses, J. Reine Angew. Math. 446 (1994) 49.

[2] A. Arnéodo, P.H. Coullet, E.A. Spiegel and C. Tresser, Asymptotic chaos, Physica D 14 (1985) 327.

[3] L. Belyakov, The bifucation set in a system with a homoclinic saddle curve, Mat. Zam. 28 (1980) 911 (in Russian).

[4] L. Belyakov, Bifurcation of systems with homoclinic curve of a saddle-focus with saddle quantity zero, Mat. Zam. 36 (1984) 681 (in Russian).

[5] A.J. Bernoff, Transitions from order in convection, Ph.D. Thesis, University of Cambridge (1986).

[6] A.R. Champneys and Yu.A. Kuznetzov, Numerical detection and continuation of codimension-two homoclinic bifurcations, Int. J. Bifurc. and Chaos 4 (1994) 785.

[7] E.J. Doedel and J.P. Kernevez, AUTO: Software for continuation and bifurcation problems in ordinary differential equations. Report, Applied Mathematics, California Institute of Technology (1986).

[8] A.C. Fowler and C.T. Sparrow, Bifocal homoclinic orbits in four dimensions, Nonlinearity 4 (1991) 1159.

[9] P. Gaspard, R. Kapral and G. Nicolis, Bifurcation phenomena near homoclinic systems: a two-parameter analysis, J. Stat. Phys. 35 (1984) 697

[10] P. Glendinning, Subsidiary bifurcations near bifocal homoclinic orbits, Math. Proc. Cambridge Philos Soc. 105 (1989) 597.

[11] P. Glendinning, Differential equations with bifocal homoclinic orbits, Int. J. Bifurcation and Chaos, to appear.

[12] P. Glendinning and C. Laing, A homoclinic hierarchy, Phys. Lett. A 211 (1996) 155.

[13] P. Glendinning and C. Sparrow, Local and global behaviour near homoclinic orbits, J. Stat. Phys. 35 (1984) 645.

[14] L.P. Shil'nikov, The existence of a denumerable set of periodic motions in a four-dimensional space in an extended neighbourhood of a saddle-focus, Sov. Math. Dokl. 8 (1967) 54

[15] L.P. Shil'nikov, A contribution to the problem of the structure of an extended neighbourhood of a rough equilibrium state of saddle-focus type, Math. USSR Sb. 10 (1970) 91.

[16] C. Sparrow, The Lorenz Equations: Bifurcations, Chaos, and Strange Attractors, Springer-Verlag Applied Mathematical Sciences, Vol. 41 (Springer, Berlin, 1982).

[17] S. Wiggins, Introduction to Applied Nonlinear Dynamical Systems and Chaos, Springer-Verlag Texts in Applied Mathematics, Vol. 2 (Springer, Berlin, 1990). 